\title{
Notes on an Information Model for Production Rules Exchange
}

\author{
Peter Denno, \\ National Institute of Standards and Technology, \\ Gaithersburg, Maryland, 20899 \\ peter.denno@nist.gov
}

\begin{abstract}
Production rules are rules used in production systems, sometimes called production rule engines. Production rule engines are commonly used to implement expert systems. This report describes an information model for the exchange of production rules and for description of the relationship of the rules to a data population. The report is a precursor to a standardization effort in the ISO 10303 suite of standards. The report takes the form of a standards document generally, but departs from that form in places to provide more userfriendly explanation.
\end{abstract}

\subsection{Scope}

This report provides guidance on two forms of data structure mapping: (1) mapping of production rules to EXPRESS-based data [1], and; (2) mapping of instances in an EXPRESS population to structures typically found in production rule systems. The EXPRESS information model which this report describes is provided in an appendix to this report. The information model provides:

- information describing rules for their use with typical production rule software

- information identifying the entity data type instances of a given EXPRESS population, and their attributes, that are subject to the rules

- information identifying the disposition of the rules with respect to the organization that produced them

- information providing logical grouping of rules

- information identifying the production rule software, and its version, with which the rules are intended to be used

- constraints concerning the form of rules

Out of scope for the information model are:

- provisions describing the syntax of rules with respect to particular production rule software

- provisions describing the execution semantics of rules 


\subsection{Definitions}

- atomic formula - a syntactic structure consisting of a predicate symbol of some arity $n$, and an $n$-tuple of terms

- condition - an atomic formula, or negated atomic formula, in the premise of a rule

Note: conditions serve as patterns used in the pattern matching process of a production rule engine

- constant - a symbol that invariantly names a distinct object

- function - a term-forming relation that maps from an n-tuple of argument to at most one object

- ground fact - an atomic formula that is free of variables

- interpretation - the assignment of objects from the domain to the appropriate constructs of a formal language

Note: These assignments are made by a human.

- object - the thing referred to by a symbol in an interpretation

- population mapping - a mapping of data governed by an EXPRESS schema to working memory of a production rule engine

- predicate - a sentence-forming relation among objects Note: A predicate is encoded as an atomic formula. A predicate (father-of henry peter) might read "Henry is the father of Peter."

- predicate symbol - a name that refers to the form of a predicate

- production memory - the internal representation of rules in a production rule system

- row value - a sequence of values, none of which are row values

- row variable - a variable naming a row value in the context of a substitution

- substitution - a mapping from variables to terms

- symbol - an atomic lexical structure

- term - an expression denoting an object

- variable - a symbol that, in the context of a substitution, names an object

- working memory - the internal representation of asserted facts in a production rule system 


\subsection{Fundamental Concepts}

\subsection{Two Targets of the Mapping}

As stated above, this report describes two forms of mapping: (1) mapping of production rules to EXPRESS-based data, and; (2) mapping of EXPRESS data types, and instances in an EXPRESS population, to three structures typically found in production rule systems: symbols, row values, and ground facts. The purpose of the first form of mapping is to allow production rules and related data to be exchanged by EXPRESSbased software tools. The purpose of the second form of mapping is to describe relationships between EXPRESS-based populations and the rules. The mapping defines how the extents of predicates can be populated so that there is an interpretation where all the rules are true.

Mapping of the production rules to the exchange form is described in Clause 3.2. Mapping of EXPRESS-based data to production rule system structures described in Clause 3.3.

EXAMPLE 1: A CLIPS [5] rule is described by data conforming to this report using the form of mapping (1), mapping rules to EXPRESS-based data.

EXAMPLE 2: A property defined by an attribute of an entity instance is mapped to a ground fact using the form of mapping (2), mapping EXPRESS-based data to production rule system structures.

EXAMPLE 3: An EXPRESS aggregate value is mapped to a production rule row value using form of mapping (2). The row value is a substitution for a row variable in the premise of a rule.

\subsection{Mapping Rule System Objects to EXPRESS-based Data}

Objects are those things that a rules engine allows to play roles in the terms of predicates. The range of such things may exceed the scope of provisions for object identification described in this report. Term_select comprises types of objects that may serve as terms in predicates.

TYPE Term_select $=$ SELECT (Symbol, Constants, Func, Rule_variable, Row_Variable); END_TYPE; 


\subsubsection{Primitive Types}

String, number, and logical values of the rule system (rule system 'constants') are mapped to their respective EXPRESS types, STRING, NUMBER, and LOGICAL.

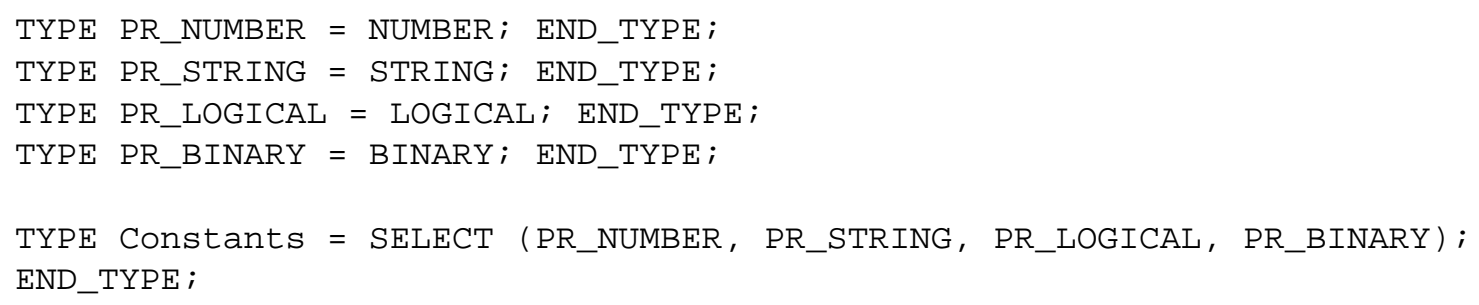

\subsubsection{Symbols}

Symbols are used by the rules system to name the things on which assertions are made. Symbols are mapped to the entity type Symbol.

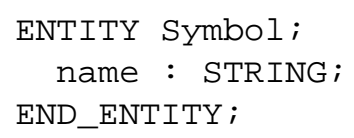

Example: EXPRESS entity instances could be represented by symbols. An entity instance encoded using the clear text encoding (ISO 10303-21) [6] \#34=POINT $(1.0,2.0,1.0)$; might be represented in the production system as a Symbol with name ' $\# 34$ '.

\subsubsection{Row values}

Row values are available in some production rule systems. Row values are mapped to Row_value.

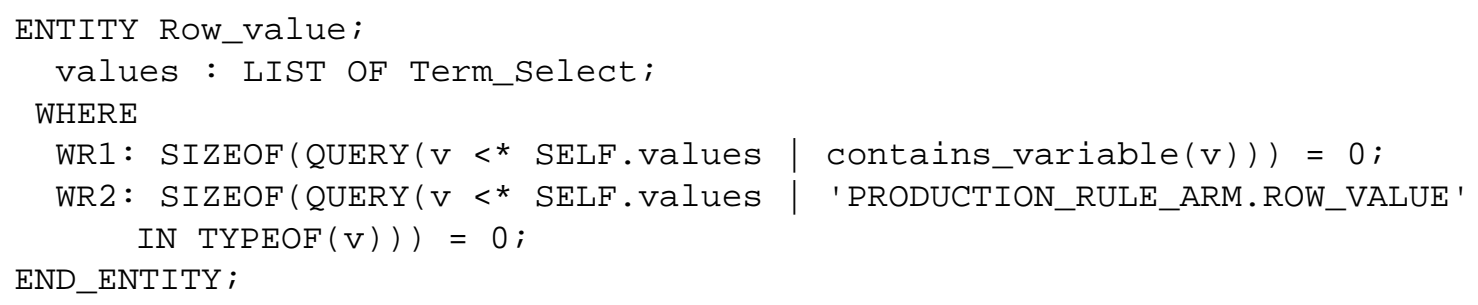

\subsubsection{Functions}

The entity data type Func is used to represent the notion of function in the production rule system.

ENTITY Func; 


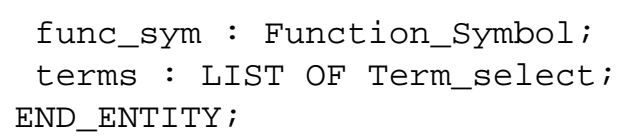

\subsubsection{Variables}

Kinds of variables are distinguished by their scope and the kind of value that they may name. Production rule systems vary with respect to their provisions for grouping rules for execution. This specification provides for grouping by Rule_set, which identifies a collection of rules, and Rule_set_group, which identifies a collection of rule sets. Variable may be scoped to individual rules, a rule set, or a rule group. A row variable is a variable naming a row value in the context of a substitution.

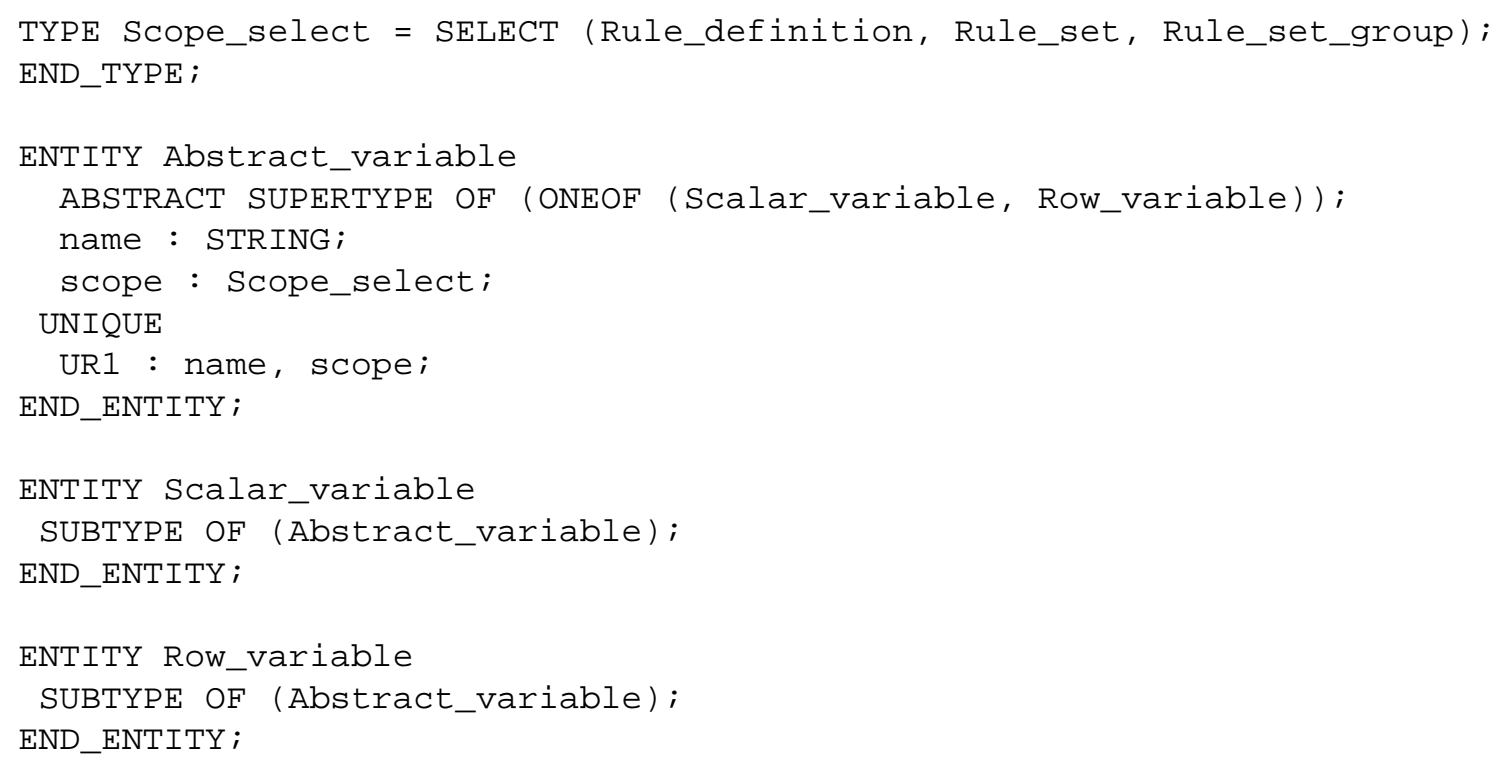

\subsubsection{Atomic Formulas and Conditions}

An atomic formula is a syntactic structure consisting of a predicate symbol of some arity $n$, and an $n$-tuple of terms. An atomic formula represents a predicate, a sentence-forming relation among the values represented by the $n$ terms. The subtype Rule_condition of entity data type Atomic_formula is used to specify a condition of a rule.

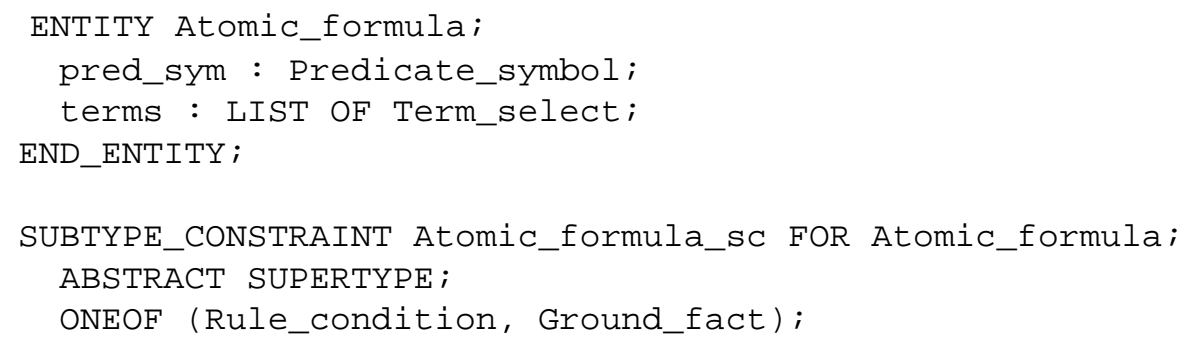




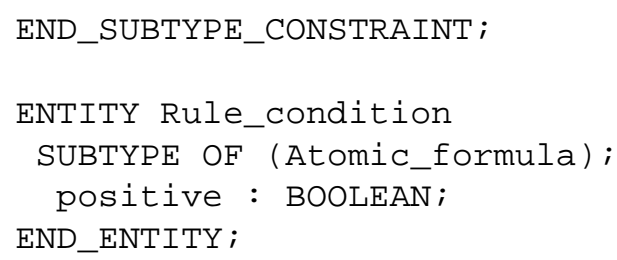

The attribute positive indicates the relationship of working memory to the satisfaction of the condition. When positive is TRUE, the condition is satisfied if a working memory element is found that matches the pattern in the context of some variable substitutions. When positive is FALSE, the condition is satisfied if no working memory element is found that matches the pattern in the context of the variable substitutions. Note that the matching is presumed to be performed in the context of variable substitutions that are consistent across all conditions of the rule.

\subsubsection{Ground Facts}

A ground fact is an atomic formula containing no variables. The entity data type Ground_fact is used to represent an item that shall be made present in the working memory of the production rules engine before the execution of rules commences.

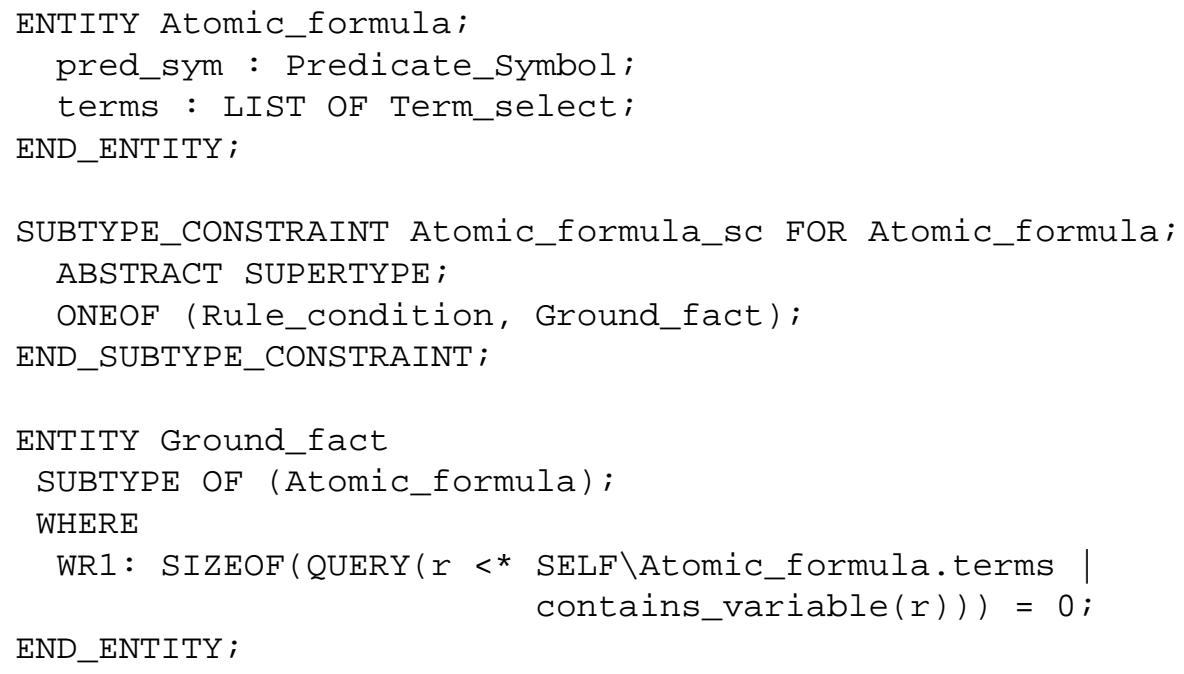

\subsubsection{Rules}

A Forward_chaining_rule represents an assertion stating that for any set of substitutions under which the premise is satisfied, the ground facts represented by the conclusion under those substitutions also hold. 
A Back_chaining_rule represents an assertion stating that for any set of substitutions under which the body is satisfied, the ground facts represented by the head under those substitutions also hold.

Forward_chaining_rule is distinguished from Back_chaining_rule by the expressions allowed in the head and body of each.

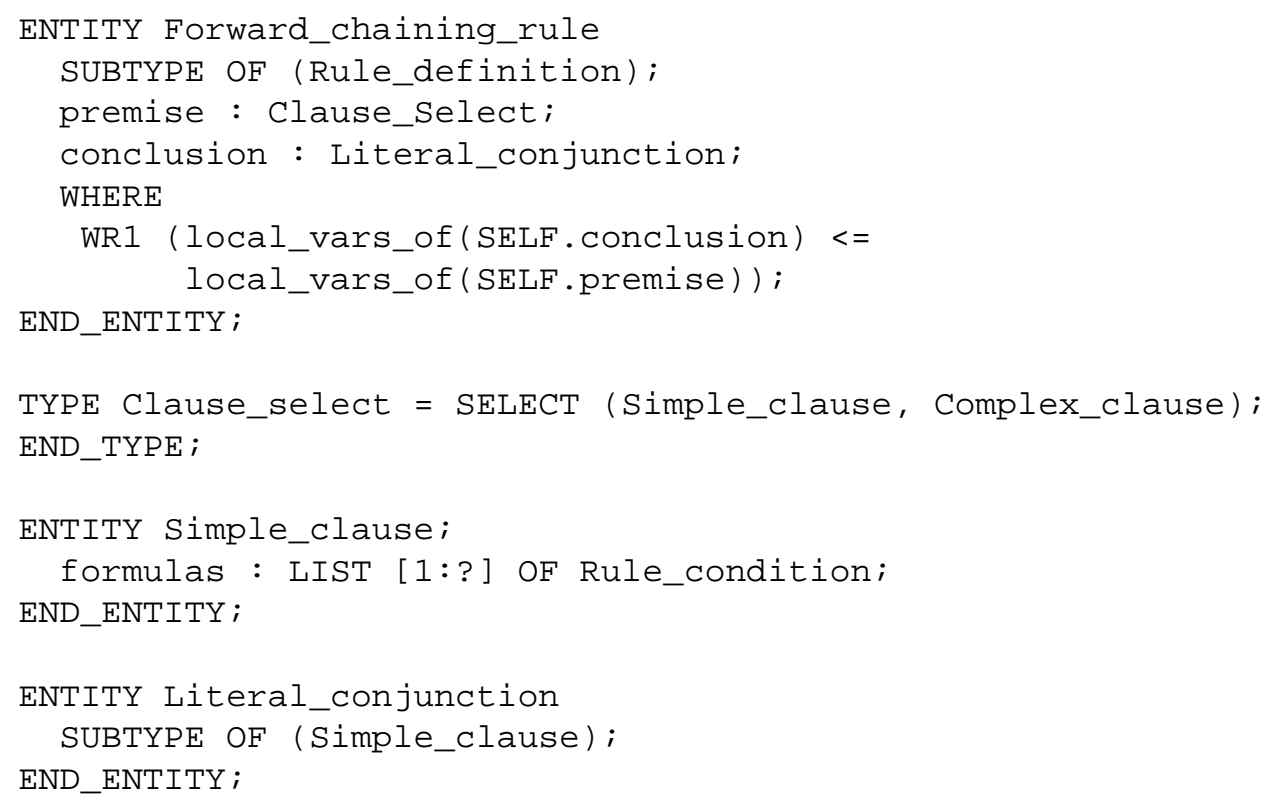

Example: The following depicts a CLIPS rule and its encoding using the clear text encoding (ISO 10303-21) [6]. The premise of the rule uses the Simple_clause syntax:

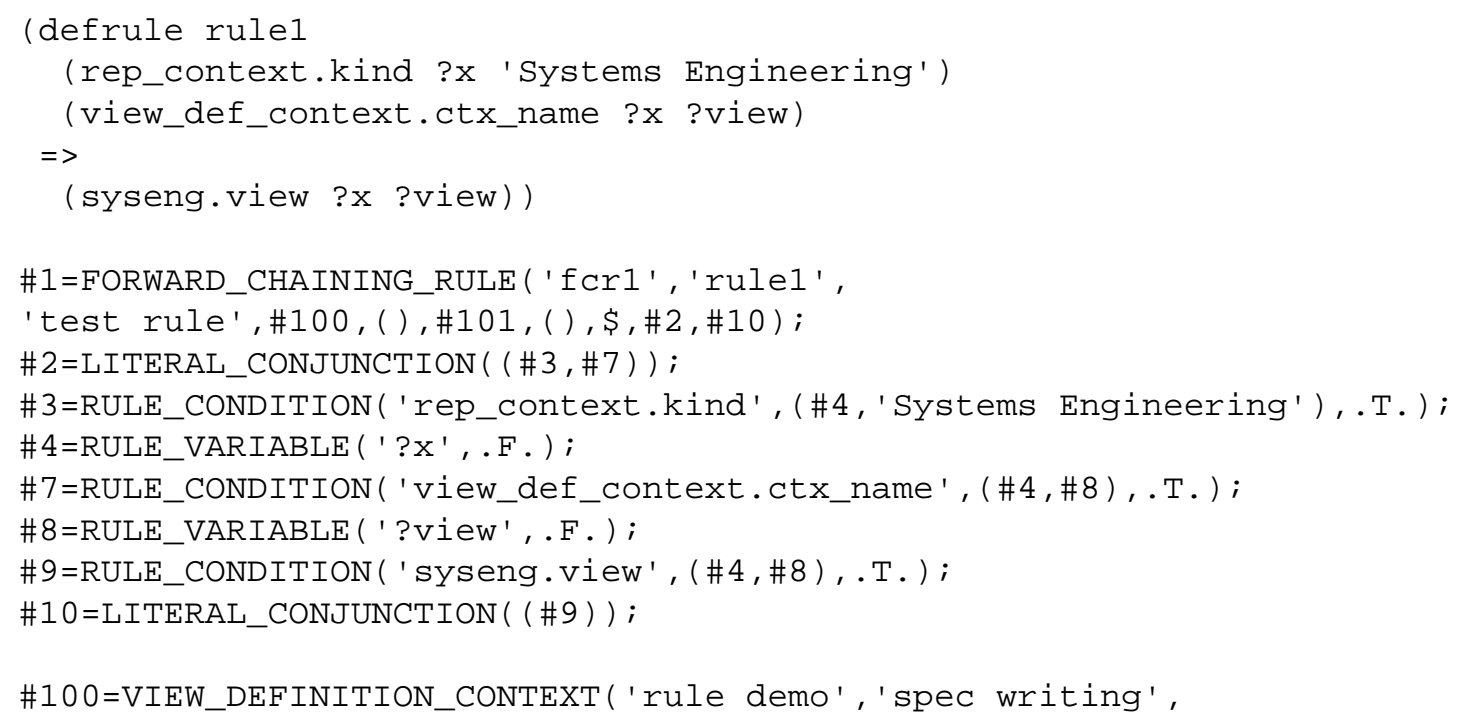


'need example for spec');

\#101=RULE_VERSION ('ver 1', 'Version 1',\#102);

\#102=RULE_PRODUCT ('rp1',' rule product 1', 'A rule product');

In the context of a substitution, a variable names an object. The object is the value of the variable under the substitution. When no substitution applies, the variable is said to be unbound. All local variables occurring in the conclusion of a rule shall also appear in the premise of a rule. The rule WR1 of Forward_chaining_rule enforces this constraint against unbound variables in the conclusion.

\subsubsection{Common Syntactic Features of Rules}

Production rule systems commonly provide syntactic features that, though they have no effect on the execution model of the system, make the formulation of rules less verbose. An example of such a syntactic convenience is the nesting of conjunctions and disjunctions of conditions in the premise of a rule. This report provides for this syntactic feature using the Complex_clause entity data type.

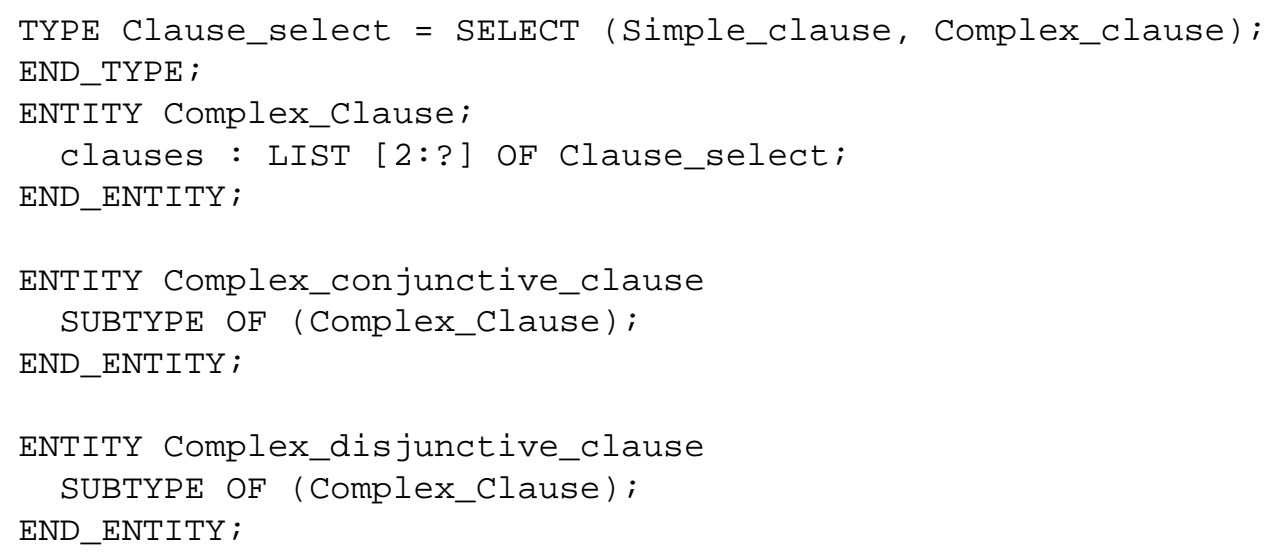

EXAMPLE: The following depicts a CLIPS rule and its encoding using the clear text encoding (ISO 10303-21) [6]. The premise of the rule uses the Complex_clause syntax:

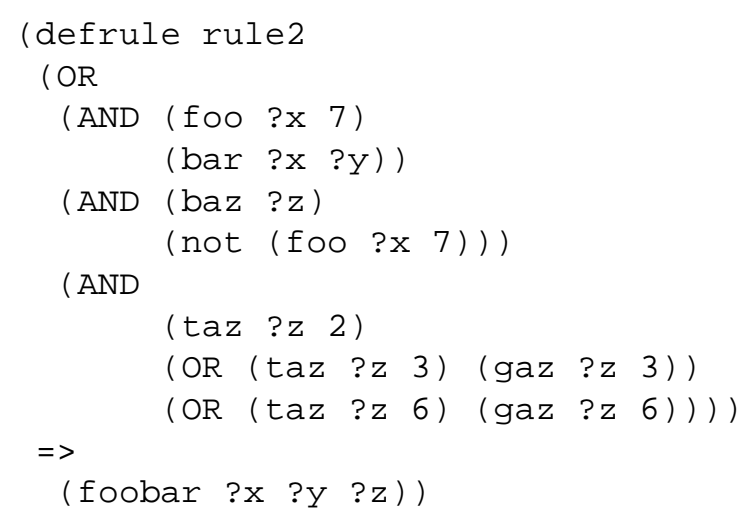




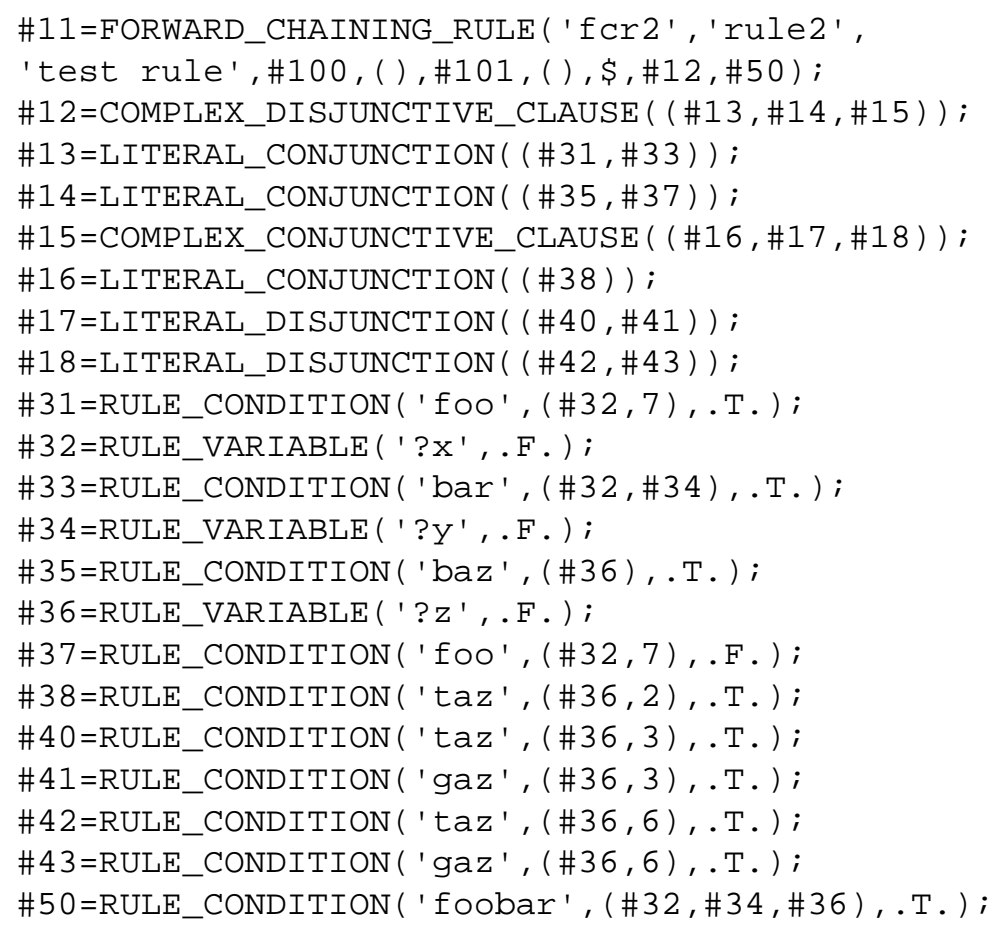

\subsection{Mapping EXPRESS-based Data to Rule System Objects}

\subsubsection{Enumeration values}

Enumeration values are mapped to symbols, where the suffix of the name of the symbol is identical with the name of the enumeration item, and the prefix of the name of the symbol are the characters specified by the prefix attribute of an instance of Enum_reference_prefix, if such an instance exists in the population. If there is no such instance, the prefix is the empty string, that is, no characters are prefixed to the name.

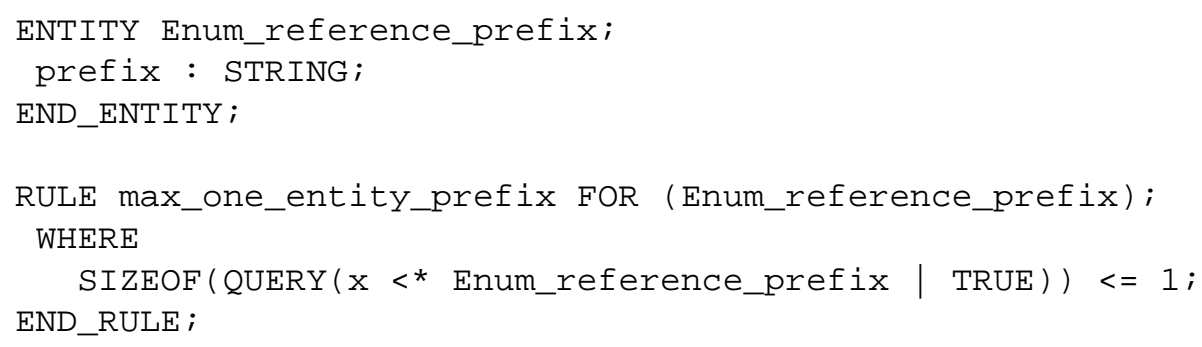

EXAMPLE: If an instance of Enum_reference_prefix is present in the population, and the value of its prefix attribute is ' : ', then an EXPRESS enumeration value GREEN is encoded as: GREEN 


\subsubsection{Aggregates}

EXPRESS aggregate values, where the base data type is not itself an aggregate, are mapped using Row_value. This report contains no provisions for mapping EXPRESS aggregates where the base data type is itself an aggregate.

\subsubsection{Selection from an EXPRESS-based population}

A population mapping is expressed through provisions that (1) identify instances involved in the mapping (described in this clause) and (2) identify what ground facts about those instances are to be asserted to working memory (described in Clause 3.3.4). Population mapping occurs before the execution of rules commences.

Instances involved in the mapping are identified as though a query were applied to a specified entity extent. The entity data type Extent provides for the expression of this.

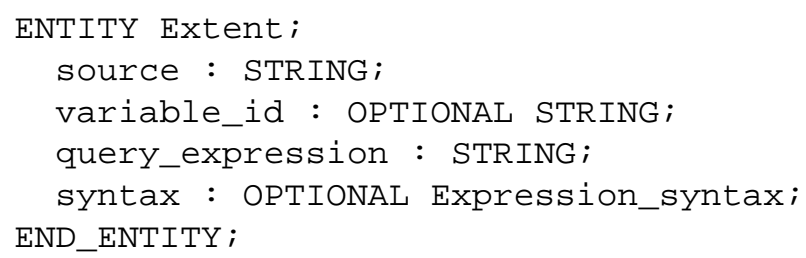

The attribute Extent . syntax indicates the syntax in which the query is specified. If the value of this attribute is EXPRESS, then instances are identified as though the EXPRESS query expression (10303-11v2 clause 12.6.7) [1] were applied to an aggregate containing a specified entity extent. The details of the mapping of the query expression to the attributes of the Extent entity type are described below.

The syntax of the EXPRESS query expression is:

QUERY '(' variable_id '<*' aggregate_source '|' logical_expression ')'

Though this syntax is not used in the mapping, it correlates with the attributes of the Extent entity:

(1) The value of Extent . variable_id performs the role of variable_id above. The value shall be a string conforming to the syntax of an EXPRESS identifier.

(2) The value of Extent .query_expression performs the role of logical_expression above. The value shall be a string conforming to the syntax of an EXPRESSS expression. The expression shall either be the EXPRESS boolean value TRUE, or it shall refer to the variable identifier supplied by Extent .variable_id. 
(3) The value of Extent . source performs the role of aggregate_source above. The value shall be the string 'GENERIC_ENTITY' or of the form 'SCHEMA.TYPE' (in upper case) where TYPE names an entity type and SCHEMA is the name of the schema that contains the definition of the type.

(4) A value shall be supplied for Extent .variable_id unless the value of Extent .query_expression is TRUE.

Under this mapping, evaluation of the query expression identifies a subset of the entity population that may be referenced as Entity_assertion. source or

Attribute_assertion.source for assertion of ground facts as described in

Clause 3.3.4. Elements taken one by one from the source aggregate provide a substitution for variable_id in the logical_expression. The logical_expression is then evaluated. If logical_expression evaluates to true, the element is added to the result; otherwise, it is not.

Example 1: If syntax is EXPRESS, an Extent instance with source 'GENERIC_ENTITY' and query_expression 'TRUE' represents an aggregate consisting of all instances in the population.

Example 2: If syntax is EXPRESS, an Extent instance with source

'my_schema.component_2d_location', variable ' $p$ ' and query_expression ' ( $p . x$ $=0)$ AND $(p \cdot y=0)$ ' represents an aggregate of all instances of type component_2d_location that are located at the origin.

Example 3: If syntax is XPATH, then query_expression might contain a W3C XPath path expression [7] that identifies a node sequence representing the intended instances.

\subsubsection{Assertion of ground facts}

Extent instances are referenced by instances of type Fact_type to represent the relationship between each element of the aggregate represented by the Extent instance and a ground fact.

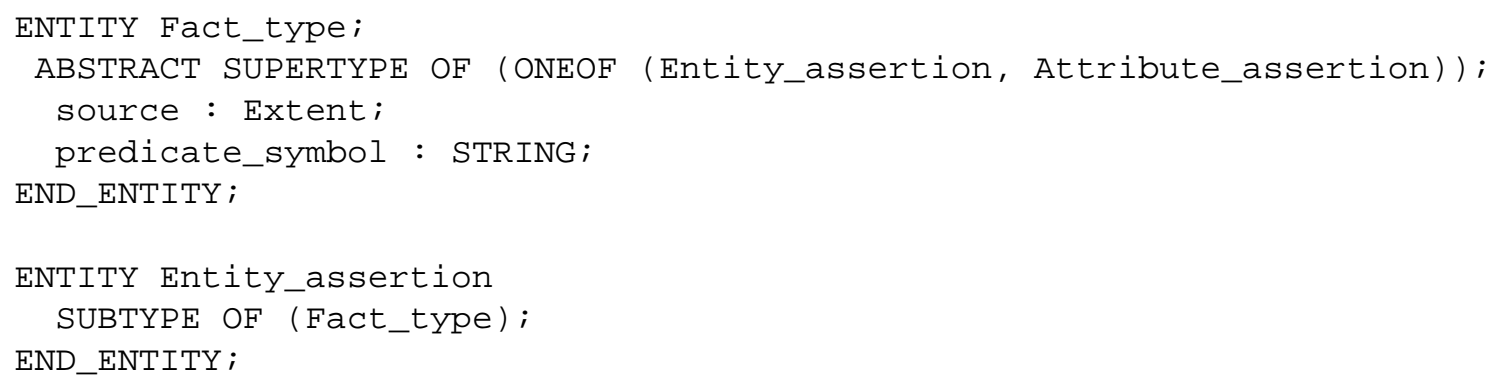

ENTITY Attribute_assertion 


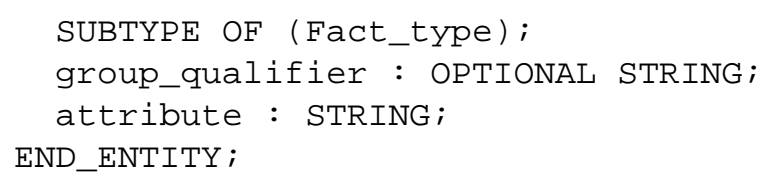

Entity_assertion represents an instruction to add one unary ground fact to working memory for each element of the aggregate represented by source. The predicate symbol of the facts asserted is that specified in attribute predicate_symbol. A ground fact is asserted for each element of the aggregate represented by source. The argument to the ground fact is a Symbol instance representing the subject element.

Attribute_assertion represents an instruction to add one binary ground fact to working memory for each element of the aggregate represented by source. The predicate symbol of the facts asserted is that specified in attribute predicate_symbol. A ground fact is asserted for each element of the aggregate represented by source. The argument to the ground fact is an ordered tuple consisting of a Symbol instance representing subject element and the value of the attribute of the instance specified by attribute, an upper case string. The value of group_qualifier shall be an upper case string naming the entity data type containing the attribute. It shall be supplied if the source of the named attribute would otherwise be ambiguous (because the complex entity instance contains multiple attributes with the name specified by attribute).

\subsection{Built-in Functions}

The Function_symbol ATTR_VAL refers a function of two arguments. The first argument shall be an entity instance and the second a string of form ENTITY. ATTRIBUTE (upper case) naming an attribute of the argument entity instance. The value of the function is the value of the named attribute of the argument instance.

EXAMPLE: Though the kind attribute of an entity Representation_context may not have been asserted as a ground fact (such as might be assumed from the example in Clause 3.2.8), a predicate may still be expressed for such a fact:

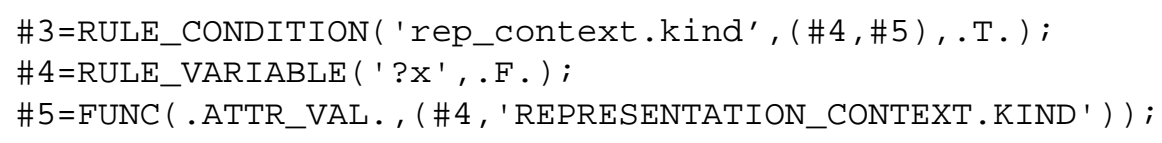

\subsection{Appendix -- EXPRESS Short Form}

SCHEMA PRODUCTION_RULE;

USE FROM Specification_document_arm; 


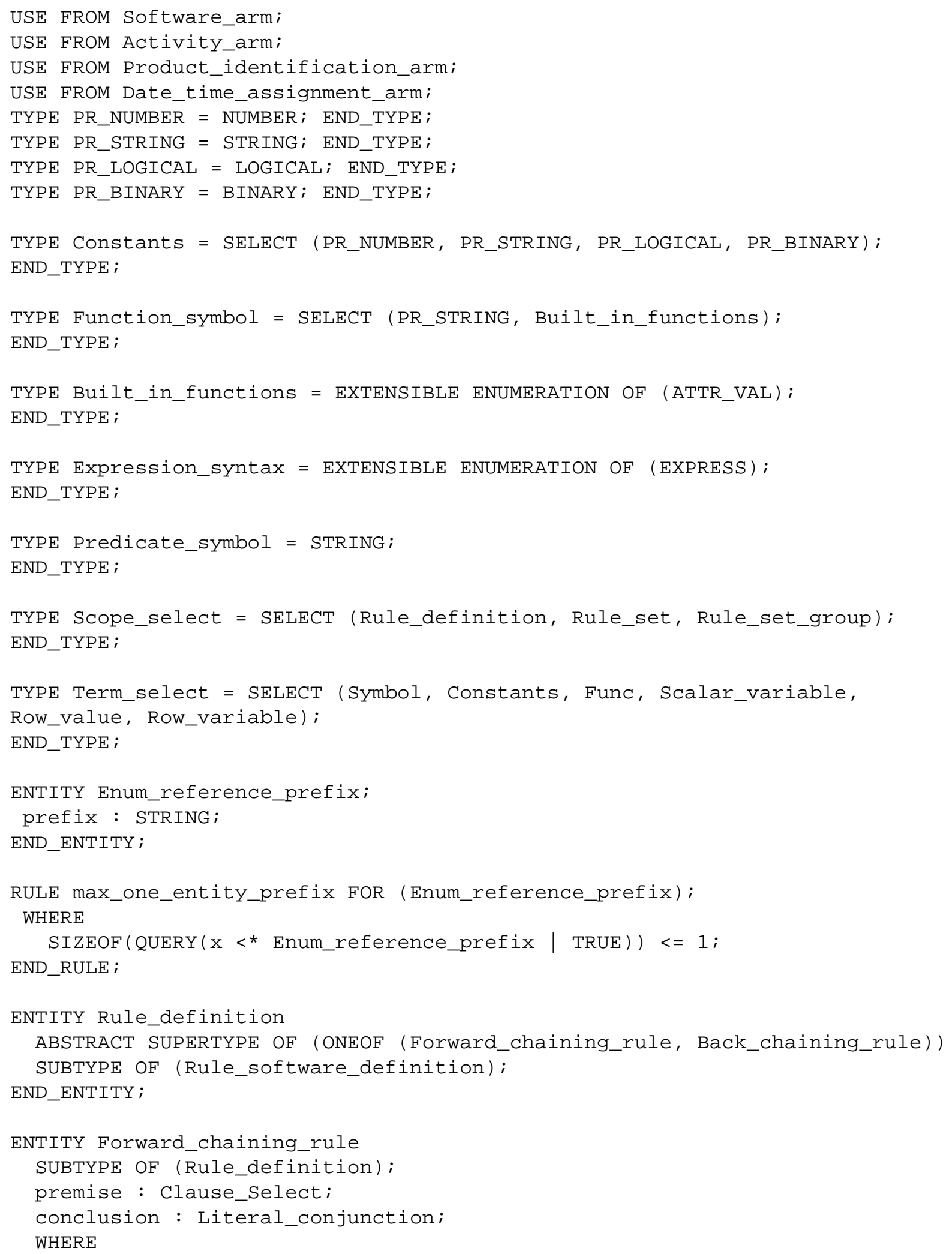




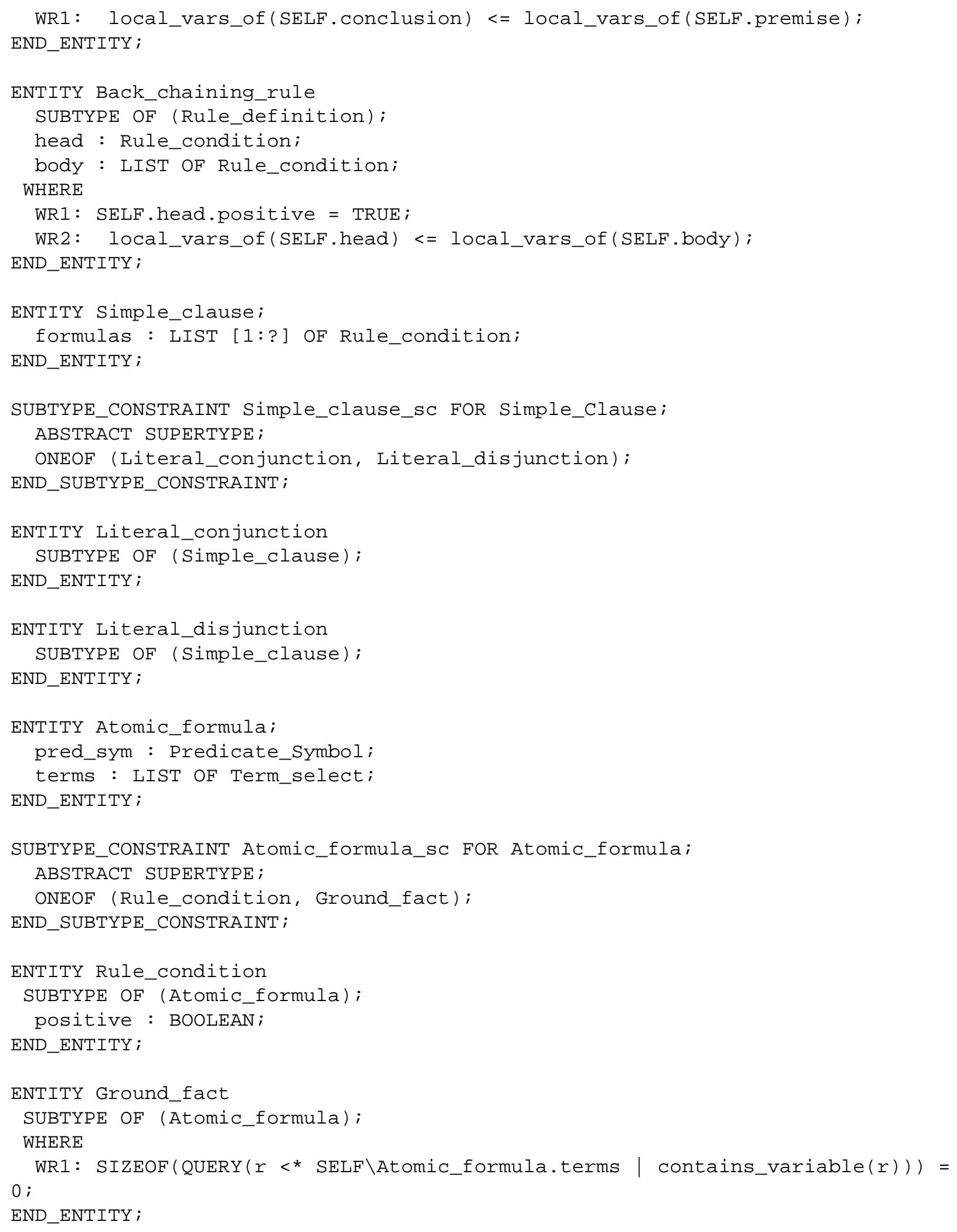




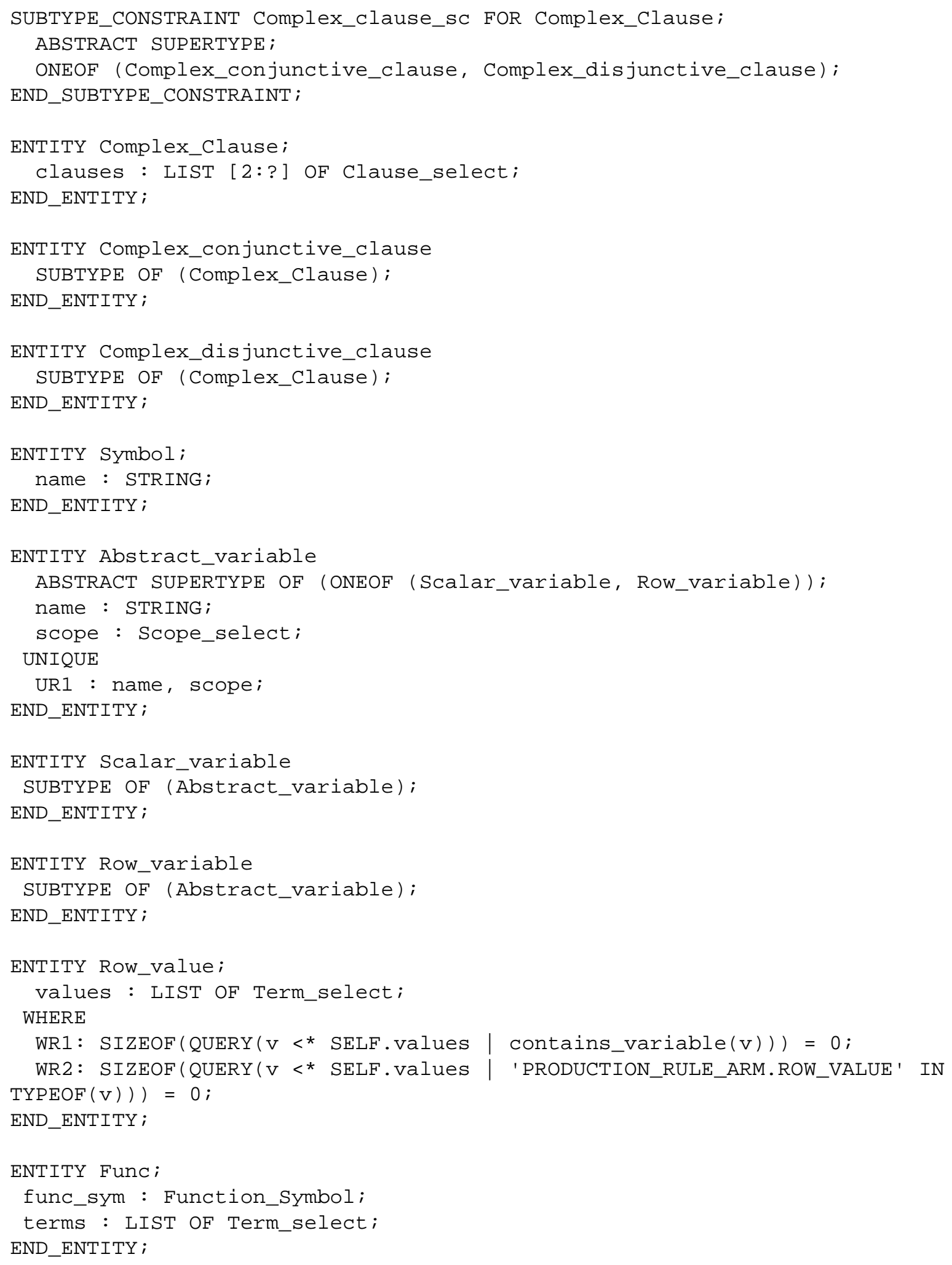




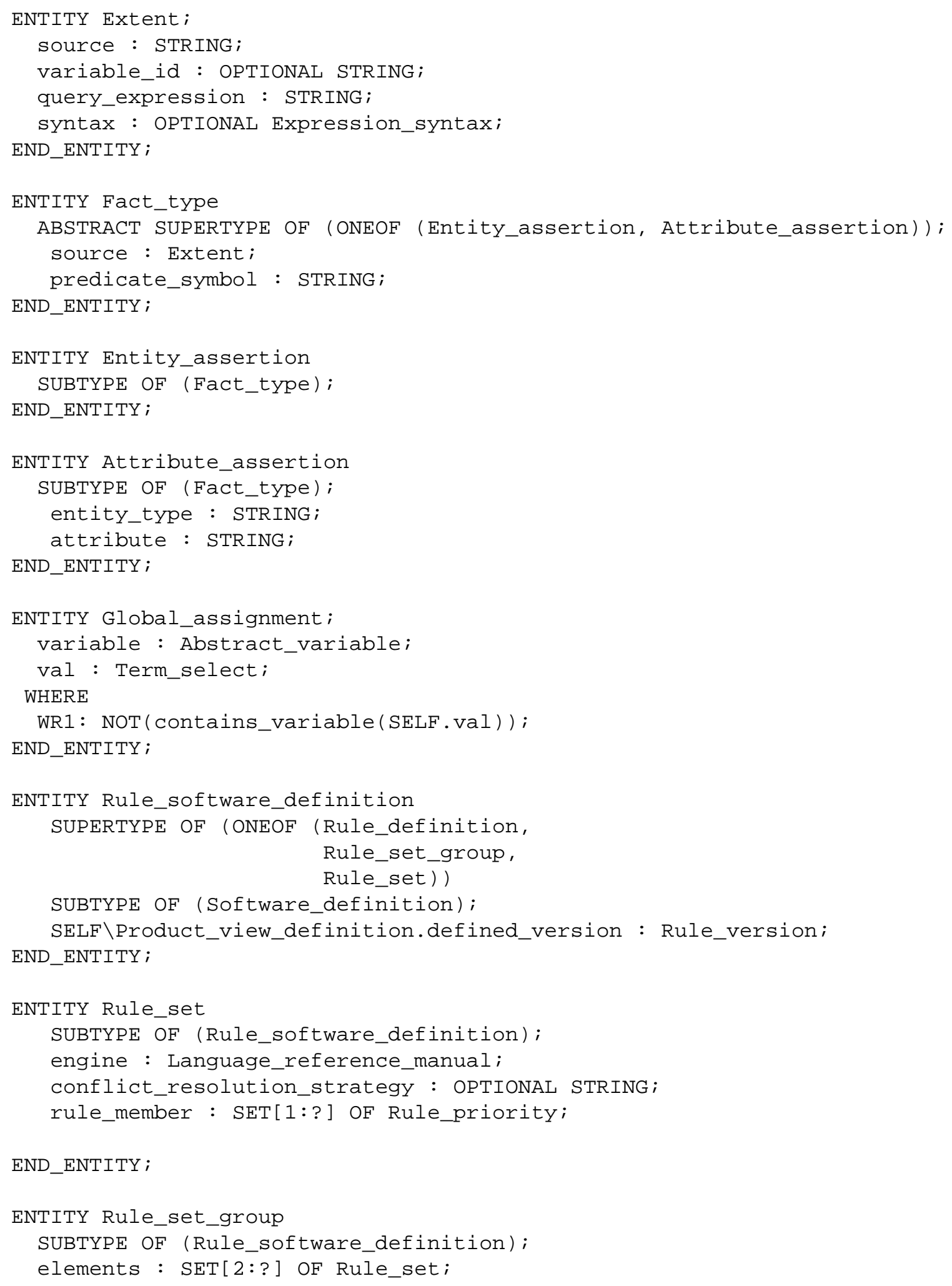




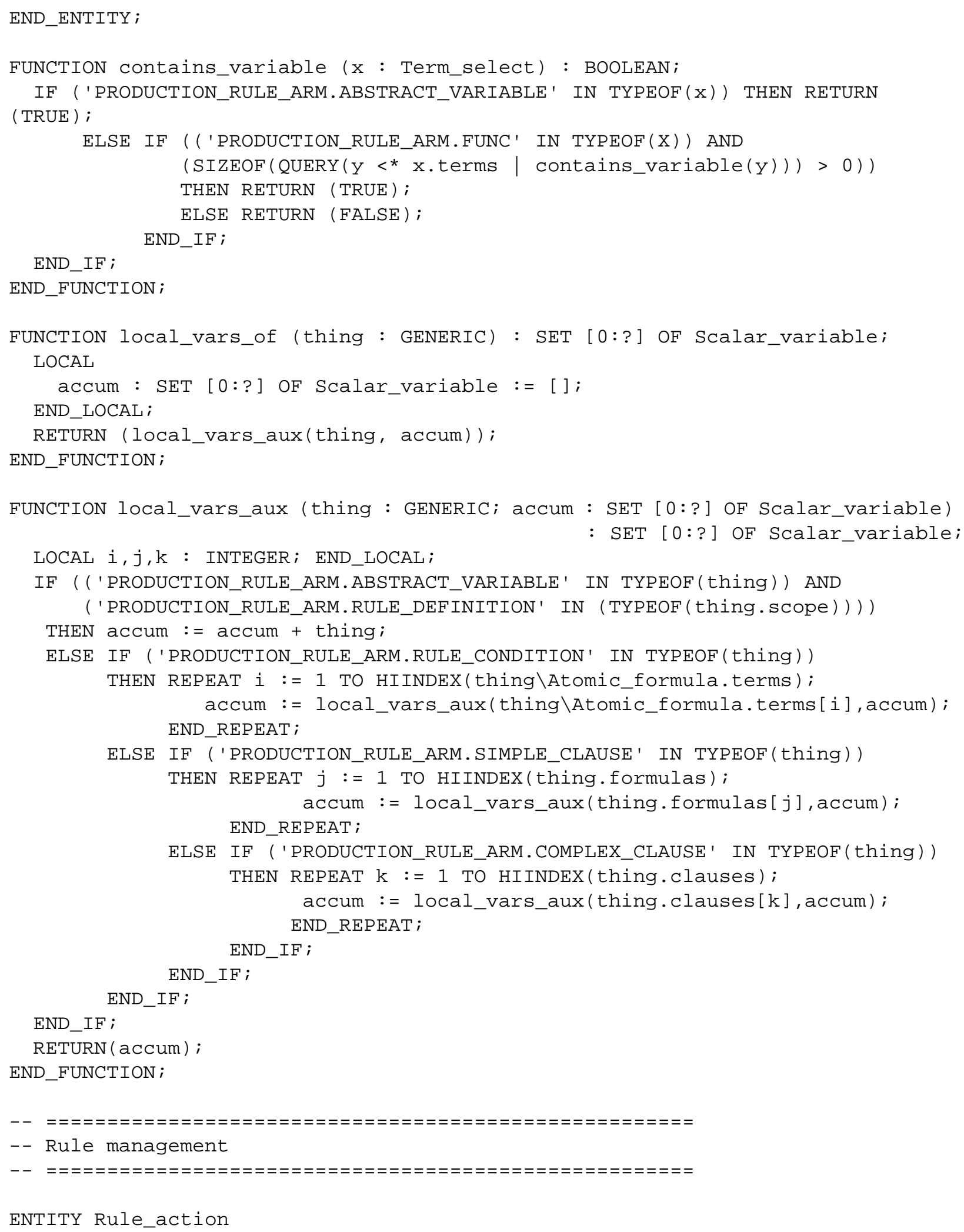

ENTITY Rule_action 


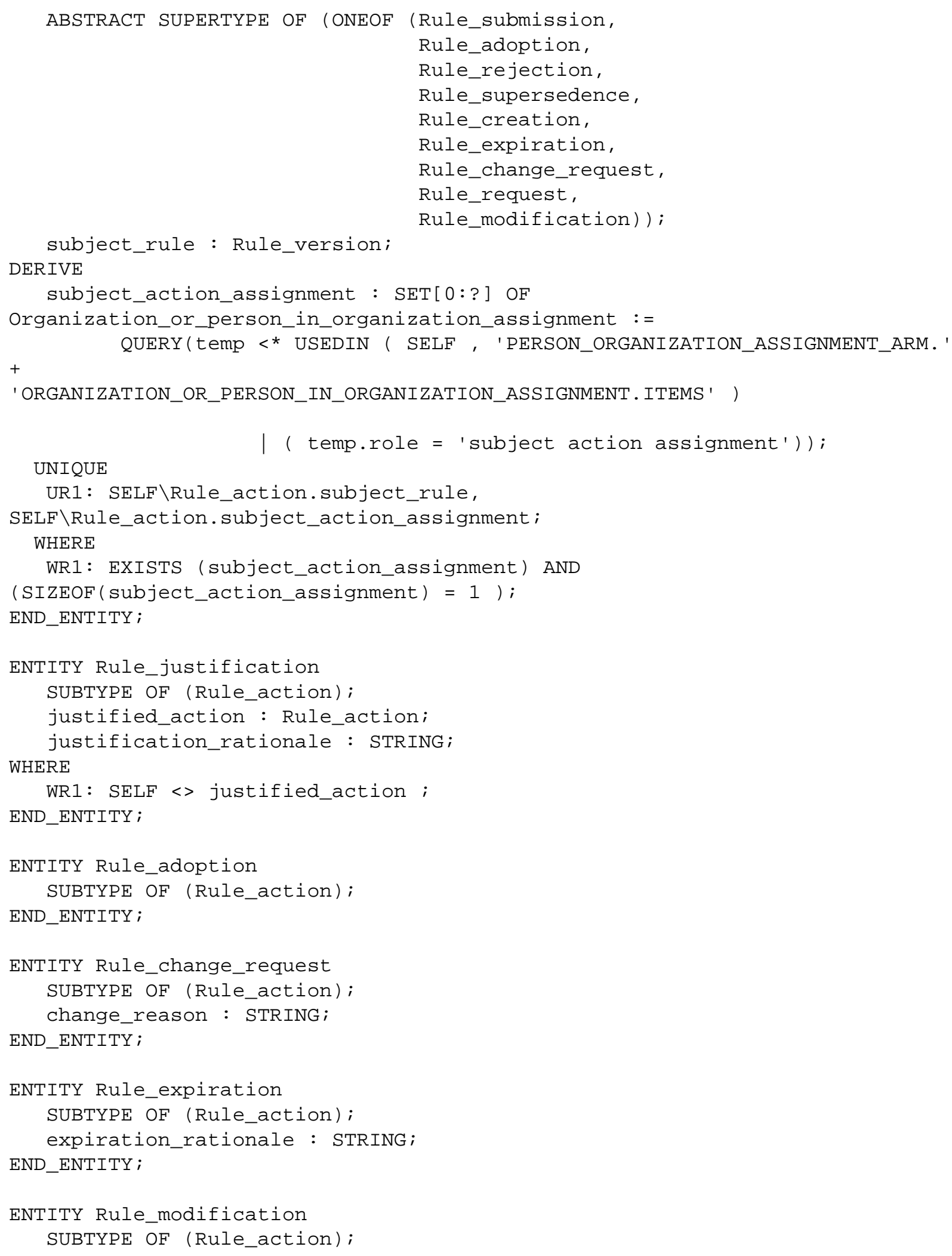




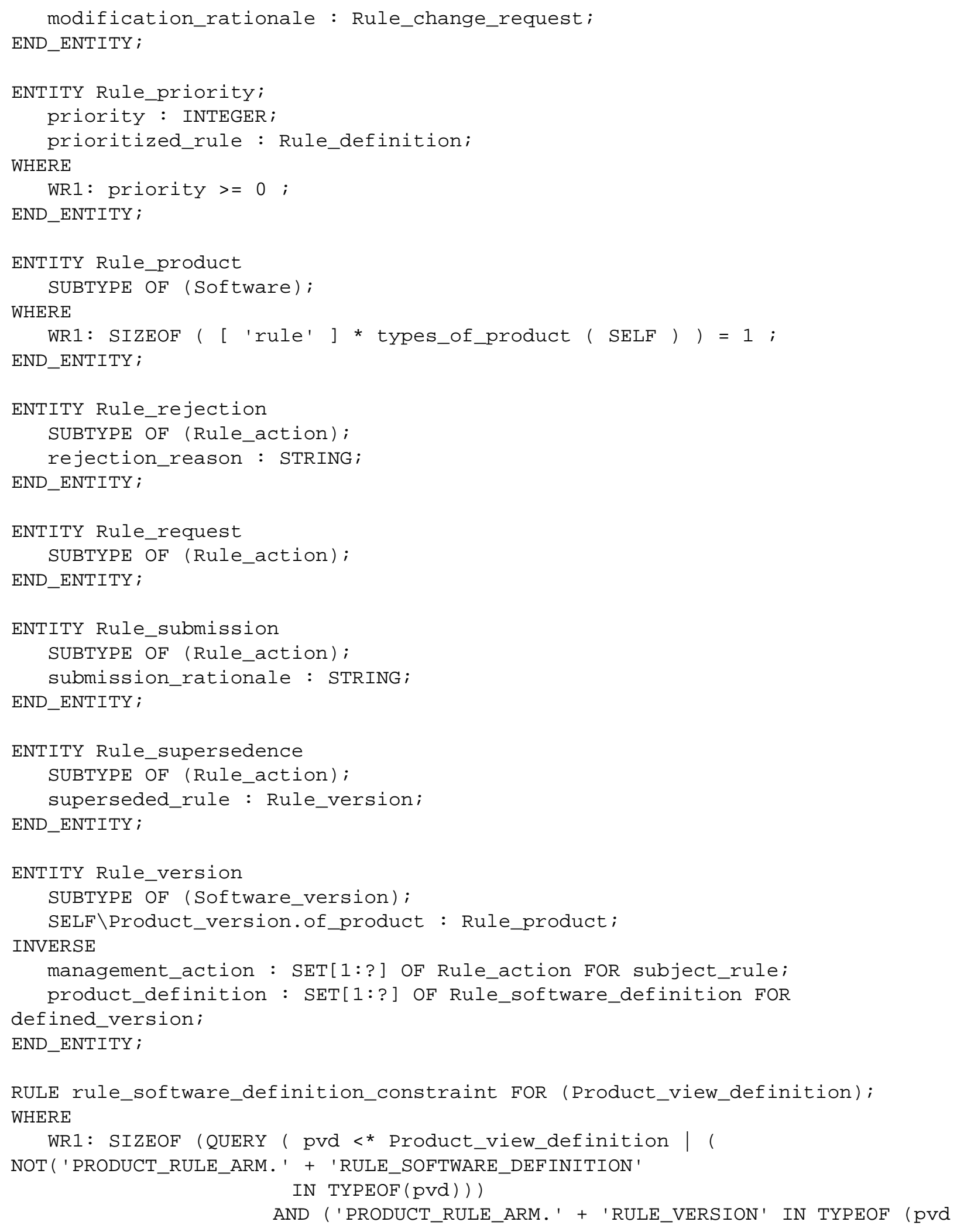




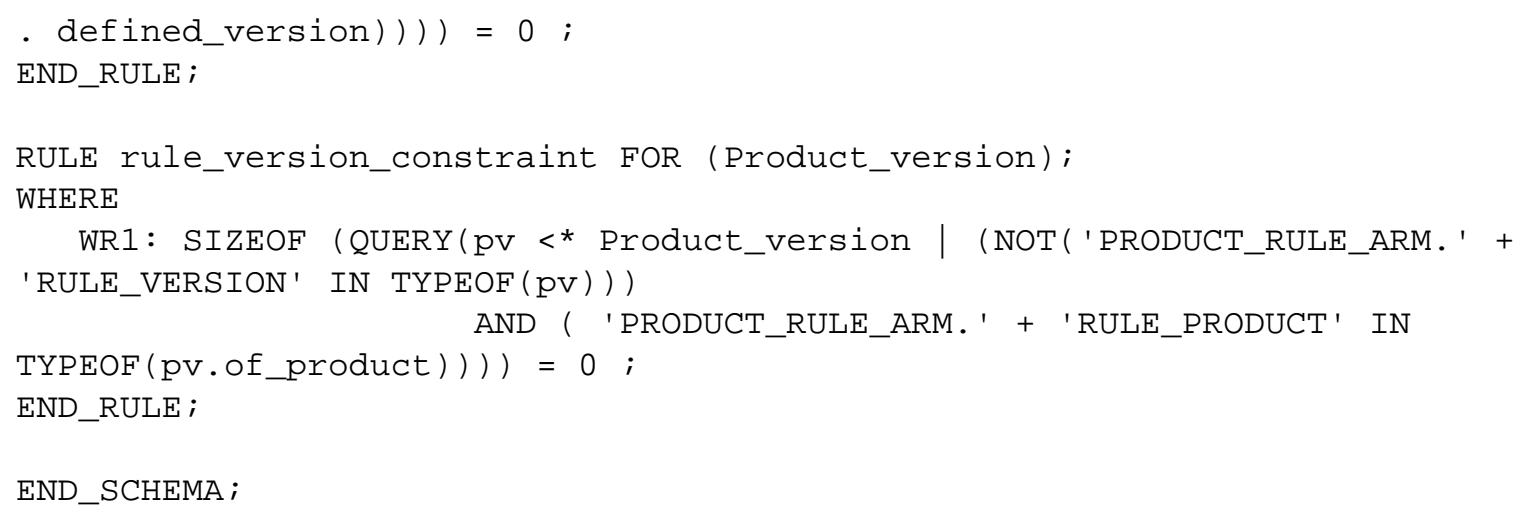

\subsection{References}

[1] International Organization for Standards, (ISO) Industrial Automation Systems and Integration - Product Data Representation and Exchange - Part 11: description methods: The EXPRESS Language Reference Manual. ISO 10303-11:2004, 2004.

[2] Robinson, J., A., and Voronkov, A., (editors) Handbook of Automated Reasoning, MIT Press, Cambridge, Massachusetts 2001.

[3] Church, A., Introduction to Mathematical Logic, Princeton University Press, Princeton, New Jersey, 1944.

[4] Doorenbos, R., B., Production Matching for Large Learning Systems, Ph.D. Dissertation, Carnegie Mellon University, 1995.

[5] unspecified authorship, CLIPS Reference Manual, Vol 1: Basic Programming Guide, Version 6.23, June 1, 2003.

[6] International Organization for Standards, (ISO) Industrial Automation Systems and Integration - Product Data Representation and Exchange - Part 21: implementation methods: Clear text encoding of the exchange structure. ISO 10303-11;1994.

[7] World Wide Web Consortium (W3C), XML Path Language (XPath), http://www.w3.org/ TR/xpath, November 16, 1999. 